\title{
Rate Coefficients of Exchange Reactions in Air and Carbon Dioxide
}

\author{
E.V. Kustova, A.S. Savelev \\ Saint-Petersburg State University, St. Petersburg, 199034 Russia \\ e.kustova@spbu.ru, aleksey.schumacher@gmail.com
}

\begin{abstract}
A simple theoretical Treanor-Marrone model is generalized to calculate state-resolved exchange reaction rate coefficients in air and carbon dioxide. An analytical dependence of the activation energy on the vibrational energy of the reactants and products is obtained for the Zeldovich reactions. This allows taking into account the vibrational excitation of NO molecules formed as a result of exchange reactions. The state-resolved reaction rate coefficients calculated using the proposed model are in good agreement with the latest data of quasi-classical trajectory calculations. The developed approach is applied to calculate the state-resolved exchange reaction rate coefficients in carbon dioxide. The effect of the model parameter on the reaction rate coefficients is estimated. Multi-temperature rate coefficients of exchange reactions in $\mathrm{CO}_{2}$ are calculated under conditions characteristic for shock waves on the basis of vibrational distributions taking into account fast intermode VV exchange between symmetric and bending modes and slow VT relaxation in bending and antisymmetric modes. The effect of vibrational excitation of various modes on the reaction rate is discussed.
\end{abstract}

Keywords: vibrational-chemical kinetics, state-to-state model, exchange reactions.

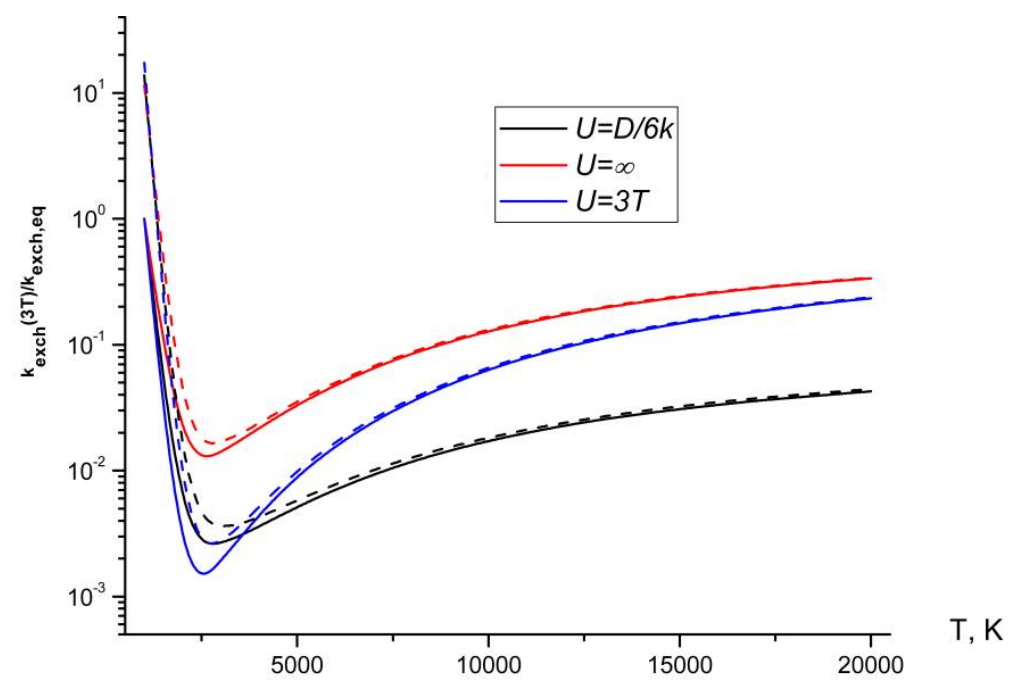

Non-equilibrium factors for multi-temperature exchange reaction rate coefficients in $\mathrm{CO}_{2}$. The solid curves are the two-temperature model at $T_{v}=1000 \mathrm{~K}$. The dashed curves are the three-temperature model with $T_{12}=1000 \mathrm{~K}$ and $T_{3}=1400 \mathrm{~K}$ 


\title{
Коэффициенты скорости обменных реакций в воздухе и углекислом газе
}

\author{
Е.В. Кустова, А.С. Савельев. \\ Санкт-Петербургский государственный университет, \\ Россия, Санкт-Петербург, 199034, Университетский наб., 7/9 \\ e.kustova@spbu.ru, aleksey.schumacher@gmail.com
}

\begin{abstract}
Аннотация
Проведено обобщение простой теоретической модели Тринора-Маррона для расчета поуровневых коэффициентов скорости обменных реакций в воздухе и углекислом газе. Для реакций Зельдовича получена аналитическая зависимость энергии активации от колебательной энергии реагентов и продуктов. Это позволило учесть колебательное возбуждение молекулы NO, образующейся в результате обменных реакций. Коэффициенты скорости реакций, рассчитанные по предложенной модели, хорошо согласуются с последними данными квазиклассических траекторных расчетов. Разработанный подход применен для вычисления поуровневых коэффициентов скорости обменных реакций в углекислом газе, оценено влияние параметра модели на скорость реакции. Проведен расчет многотемпературных коэффициентов скорости обменных реакций в $\mathrm{CO}_{2}$ в условиях, характерных для течений за ударными волнами. При этом использованы колебательные распределения, учитывающие быстрый межмодовый VV обмен между симметричной и деформационной модами и замедленную VT релаксацию в деформационной и антисимметричной модах. Обсуждается влияние колебательного возбуждения различных мод на скорость реакции.
\end{abstract}

Ключевые слова: колебательно-химическая кинетика, поуровневая модель, обменные реакции.

\section{1. Введение}

Современные вычислительные возможности позволяют использовать при решении задач высокотемпературной и высокоэнтальпийной газовой динамики не только привычные многотемпературные модели колебательно-химического взаимодействия, но и более точные, поуровневые. Однако далеко не все используемые методы позволяют получить высокую точность результатов без значительного усложнения вычислительных кодов. Это приводит нас к необходимости построения моделей, объединяющих в себе простоту реализации и интеграции в существующие коды, высокую скорость расчета, хорошее согласование коэффициентов скорости реакций с данными траекторных расчетов.

Целью данного исследования является обобщение широко известной модели ТринораМаррона [1] для расчета поуровневых коэффициентов скорости обменных химических реакций в воздухе и в углекислом газе. На первом этапе, основываясь на сравнении с данными квазиклассических траекторных расчетов, была построена модель, учитывающая колебательное возбуждение продуктов реакций Зельдовича в воздухе. Результаты проверяются сравнением с данными современных траекторных расчетов.

Далее, используя результаты, полученные для воздуха, рассматривается задача о вычислении поуровневых и многотемпературных коэффициентов скорости обменных химических реакций в смесях, содержащих углекислый газ и продукты его диссоциации, изучается влияние выбора параметров модели на результаты расчета коэффициентов. 


\section{2. Поуровневые коэффициенты скорости обменных реакций в воздухе с учетом колебательного возбуждения пробукта реакции}

Наряду с реакциями диссоциации, обменные реакции играют важную роль в описании процессов неравновесной кинетики, однако их зависимость от колебательных уровней реагентов изучена довольно слабо. В литературе встречаются несколько способов определения данных коэффициентов: траекторные расчеты $[2,3,4]$, обобщения модели Тринора-Маррона для обменных реакций $[5,6,7,8]$, данные, полученные в результате аппроксимации траекторных расчетов Бозе-Кандлера [9, 10], экспериментальные данные [11]. Некоторые результаты квазиклассических траекторных расчетов также доступны в базе данных STELLAR [12]. Недавние результаты расчетов ab initio с учетом вращательных состояний представлены в работах $[13,14]$.

В настоящей работе исследуются поуровневые реакции Зельдовича

$$
\begin{aligned}
& \mathrm{N}_{2}(i)+\mathrm{O} \rightarrow \mathrm{NO}+\mathrm{N}, \\
& \mathrm{O}_{2}(i)+\mathrm{N} \rightarrow \mathrm{NO}+\mathrm{O}
\end{aligned}
$$

В предшествующих работах $[15,16]$ было проведено исчерпывающее сравнение существующих теоретических моделей с результатами квазиклассических траекторных расчетов и показано, что, для основного колебательного состояния продукта реакции, наилучшее совпадение достигается при использовании исправленной модели Алиата [8]. Существенным же недостатком большинства существующих моделей является невозможность учета колебательного состояния продукта реакции. В то же время данные, учитывающие возбуждение продукта, представлены в последние годы сразу несколькими группами, занимающимися траекторными вычислениями $[13,14,17,18]$. Сравнение представленных данных из [12] и [14] позволяет сделать вывод об их обоснованности и возможности использования в качестве эталонных при построении модели, учитывающей колебательное возбуждение продукта реакции. Для этих целей мы использовали данные [12], ввиду их большей гладкости и исправленную модель Алиата [8] в качестве теоретической основы. Для этой модели поуровневые коэффициенты скорости обменных реакций для основного электронного состояния реагента представляются в виде:

$$
k_{M_{2} i}^{\text {exch }}(T, U)=\left\{\begin{array}{r}
C(T, U) k_{e q}^{\text {exch }} \exp \left(-\frac{E_{a}}{k U}\right) s_{i} \exp \left[\frac{\varepsilon_{i}^{M_{2}}}{k}\left(\frac{1}{T}+\frac{1}{U}\right)\right], \varepsilon_{i}^{M_{2}}<E_{a} \\
C(T, U) k_{e q}^{\text {exch }} \exp \left(\frac{E_{a}}{k T}\right), \varepsilon_{i}^{M_{2}}>E_{a}
\end{array}\right.
$$

Нормирующий множитель определяется соотношением

$$
C(T, U)=Z_{M_{2}}^{v i b r}(T)\left[\sum_{i=0}^{i^{*}} s_{i} \exp \left(-\frac{E_{a}-\varepsilon_{i}^{M_{2}}}{k U}\right)+\sum_{i^{*}+1}^{l_{S}} s_{i} \exp \left(\frac{E_{a}-\varepsilon_{i}^{M_{2}}}{k T}\right)\right]^{-1}
$$

Здесь $T$ - температура газа; $k$ - постоянная Больцмана; $E_{a}-$ энергия активации; $Z_{\mathrm{M}_{2}}^{v i b r}(T)-$ равновесная колебательная статистическая сумма молекулы-реагента; $M_{2}$ и $i$ означают химический сорт и колебательное состояние реагирующей молекулы; $\varepsilon_{i}^{M_{2}}, s_{i}$ - колебательная энергия и статистический вес соответствующего состояния. Для двухатомного газа $s_{i}=1$. $i^{*}$ обозначает последний колебательный уровень, энергия которого меньше энергии активации реакции $\varepsilon_{i}^{M_{2}}<E_{a} ; l_{s}$ отвечает за номер последнего колебательного уровня с энергией ниже энергии диссоциации сорта $M_{2} ; k_{e q}^{e x c h}$ - равновесный коэффициент скорости обменной реакции, вычисляемый по закону Аррениуса:

$$
k_{\text {exch,eq }}^{M}(T)=A T^{b} \exp \left(-\frac{E_{a}}{k T}\right)
$$

$A, b$ - параметры, получаемые из экспериментальных данных $[9,10]$. 
Параметр модели $U$ имеет размерность температуры; его физический смысл, как и в оригинальной модели Тринора-Маррона, заключается в учете увеличения вероятности реакции для колебательно возбужденных состояний. Значение параметра $U=\infty$ соответствует равной вероятности реакции из любого состояния.

Как уже было отмечено, большинство существующих теоретических моделей позволяют вычислить коэффициенты скорости обменных реакций только для основного энергетического состояния NO. Исключением является только модель, представленная в работе [18]. Наши предыдущие исследования $[15,16]$ продемонстрировали важность учета колебательного состояния продукта реакции при моделировании течений за фронтом ударной волны. В связи с этим и, принимая во внимание появление в литературе результатов высокоточных траекторных расчетов $[12,14]$, мы решили модернизировать модель Алиата для учета колебательного возбуждения продукта реакции.

В результате анализа траекторных данных $[12,14]$ было установлено, что учет возбуждения продукта инициирует сдвиг порога активации реакции к более высоким колебательным состояниям реагента на величину, сопоставимую с колебательной энергией возбужденного состояния продукта реакции. При этом наилучшее согласие с траекторными данными достигается в результате использования в качестве параметра модели $U=\infty$. В результате итоговый вид формулы получается

$$
k_{M_{2}(i), \mathrm{NO}(k)}^{\text {exch }}(T, U)=\left\{\begin{array}{l}
C_{k}(T) k_{e q, k}^{\text {exch }} \exp \left(\frac{\varepsilon_{i}^{M_{2}}}{k T}\right), \varepsilon_{i}^{M_{2}}<E_{a}(k) \\
C_{k}(T) k_{e q, k}^{\text {exch }} \exp \left(\frac{E_{a}(k)}{k T}\right), \varepsilon_{i}^{M_{2}}>E_{a}(k)
\end{array}\right.
$$

Нормирующий множитель имеет вид

$$
C_{k}(T)=Z_{M_{2}}^{v i b r}(T)\left[i^{*}+1+\sum_{i^{*}+1}^{l_{s}} \exp \left(\frac{E_{a}(k)-\varepsilon_{i}^{M_{2}}}{k T}\right)\right]^{-1}
$$

Следует отметить, что в обобщенной модели энергия активации является функцией колебательного состояния продукта реакции. Кроме того, закон Аррениуса также требует модификации и записывается в форме

$$
k_{e q, k}^{e x c h}(T)=A P\left(\varepsilon_{k}^{\mathrm{NO}}\right) T^{b} \exp \left(-\frac{E_{a}(k)}{k T}\right)
$$

Значения параметров, используемых в уравнениях (6)-(8), представлены в работе [16]. Следует пояснить, что в [16] в таблице 1 на странице 6 допущена опечатка в первом столбце: в первой строке представлены параметры для реакции $\mathrm{N}_{2}+\mathrm{O}$, а во второй - для реакции $\mathrm{O}_{2}+\mathrm{N}$.

На рис. 1 и 2 представлено сравнение поуровневых коэффициентов скорости обменных реакций, вычисленных с помощью предложенной модели, с коэффициентами из базы данных квазиклассических траекторных расчетов (QCT) [12]. На графиках представлено сравнение коэффициентов скорости обменных реакций при фиксированной температуре от колебательного уровня реагента для разных колебательных уровней $\mathrm{NO}(k)$. Легко видеть хорошее согласование полученных результатов с данными траекторных расчетов. Анализ поведения коэффициентов скорости реакции (1) показывает, что они растут практически линейно до значений колебательной энергии реагента, соответствующей энергии $E_{a}(k)$, а затем перестают зависеть от колебательной энергии. Поведение коэффициентов скорости реакции (2) несколько отличается вследствие малости ее энергии активации и более низкого порога диссоциации молекулы кислорода по сравнению с молекулами азота и оксида азота. Кроме того, для реакции (2) и низких колебательных уровней оксида азота наблюдается нечувствительность коэффициентов скорости обменной реакции к колебательной энергии NO (в частности, на графике кривые для $k=0$ и $k=5$ совпадают). 


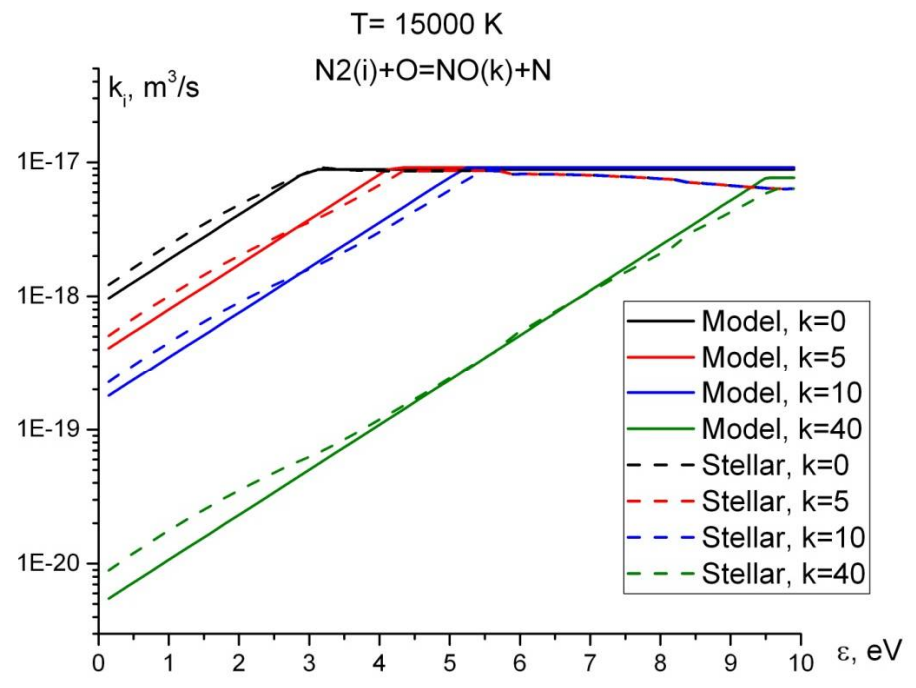

Рис. 1. Коэффициенты скорости обменных реакций как функция от колебательного уровня $\mathrm{N}_{2}$ для различных колебательных состояний NO. Реакция $\mathrm{N}_{2}+\mathrm{O} \rightarrow \mathrm{NO}+\mathrm{N}$. Сравнение траекторных расчетов [12] и представленной модели, $T=5000 \mathrm{~K}$

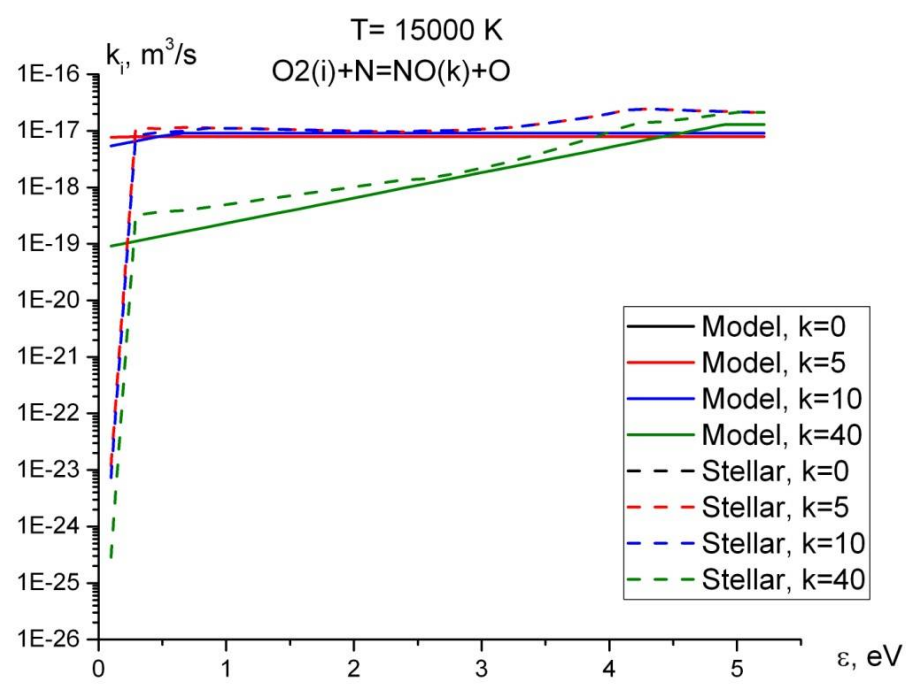

Рис. 2. Коэффициенты скорости обменных реакций как функция от колебательного уровня $\mathrm{O}_{2}$ для различных колебательных состояний NO. Реакция $\mathrm{O}_{2}+\mathrm{N} \rightarrow \mathrm{NO}+\mathrm{O}$. Сравнение траекторных расчетов [12] и представленной модели, $T=5000 \mathrm{~K}$

\section{3. Коэффициенты скорости обменных реакций в углекислом газе}

В отличие от молекул кислорода и азота, которые были рассмотрены в предыдущей части работы, молекула углекислого газа обладает гораздо более сложной структурой, что существенно увеличивает сложность ее моделирования. Другой важной проблемой является полное отсутствие данных траекторных расчетов для поуровневых коэффициентов скоростей реакции в $\mathrm{CO}_{2}$, что не дает возможности провести надежную валидацию теоретических моделей. Тем не менее, в последнее время появилась большая потребность в теоретических моделях поуровневых обменных реакций для моделирования как высокотемпературных процессов при входе в атмосферу Марса $[19,20,21]$, так и низкотемпературных плазмохимических процессов в экспериментах по конверсии углекислого газа [22, 23, 24, 25]. В работе мы 
предлагаем обобщение модели, разработанной для обменных реакций в воздухе, и первые результаты расчетов. Проверку модели предполагается провести позднее косвенными методами, при сравнении с экспериментальными данными по измеренным температурам и тепловым потокам.

\section{1. Поуровневые коэффициенты скорости обменных реакций}

Молекула углекислого газа имеет три колебательные моды: симметричную, дважды вырожденную деформационную и антисимметричную. Наличие межмодовых обменов и существенные различия в характерных временах VV и VT процессов приводят к сильной связи заселенностей колебательных уровней мод. Более того, число различных колебательных состояний молекул $\mathrm{CO}_{2}$ огромно: в случае гармонического осциллятора молекула имеет более 6000 колебательных состояний, а в случае использования модели ангармонического состояния - почти 9000 различных колебательных состояний.

Рассмотрим обменную реакцию

$$
\mathrm{CO}_{2}(i)+\mathrm{O} \rightarrow \mathrm{CO}+\mathrm{O}_{2}
$$

Здесь индекс $i$ является совокупностью трех индексов $\left(i_{1}, i_{2}, i_{3}\right)$, соответствующих уровням симметричной, деформационной и антисимметричной мод. Максимальное число уровней в каждой моде для модели гармонического осциллятора $l_{1}=33, l_{2}=65, l_{3}=19$. При расчете колебательной энергии учитываются все состояния, лежащие ниже порога диссоциации.

Как уже было отмечено, в отличие от молекул воздуха, для молекулы углекислого газа нет данных траекторных расчетов и теоретических моделей поуровневых коэффициентов реакции. Мы предполагаем, что формулы, выведенные для воздуха, будут справедливы и в случае молекулы углекислого газа. Таким образом, для обменных реакций будет использоваться исправленная модель Алиата [8] в форме (3)-(5) для основного колебательного состояния продуктов реакции. Однако, по причинам, связанным со структурой молекулы углекислого газа, в формулы необходимо ввести поправки: во-первых, статистический вес не равен единице вследствие вырождения второй колебательной моды, $s_{i 1 i 2 i 3}=i_{2}+1$. Во-вторых, суммирование ведется по трем индексам $\left(i_{1}, i_{2}, i_{3}\right)$ для всех состояний ниже энергии диссоциации. При расчете варьируется параметр модели $U$. Параметры в законе Аррениуса для реакции (9) взяты из [26] и равны: $A=2.1 \times 10^{7}, n=0, E_{a}=27800 \mathrm{~K}$.

На рис. 3 приведены коэффициенты скорости обменной реакции (9) как функции колебательной энергии молекулы $\mathrm{CO}_{2}$ для разных температур и значений параметра $U$. Легко заметить, что выбор параметра $U$ оказывает существенное влияние на величину коэффициентов во всем диапазоне температур. Разница в результатах, получаемых при использовании значений, используемых в литературе, проявляется в двух фактах. Во-первых, разница в величине уровневых коэффициентов для нижних колебательных состояний (ниже энергии активации) может достигать нескольких порядков величин. При этом точка выхода на плато совпадает с энергией активации для всех вариантов выбора параметра $U$, но сама величина плато отличается для разных значений параметра. С ростом температуры данная разница уменьшается. Поскольку для воздуха наилучшее согласие с квазиклассическими траекторными расчетами достигалось при $U=\infty$, мы полагаем, что в углекислом газе выбор значений $U=D / 6 k$ или $U=3 T$ может приводить к недооценке значений коэффициентов скорости реакции для нижних состояний. Из данного анализа можно сделать вывод о том, что правильный выбор модели и ее параметров может оказывать существенное влияние на скорость обменных реакций в углекислом газе, особенно при низких температурах. 

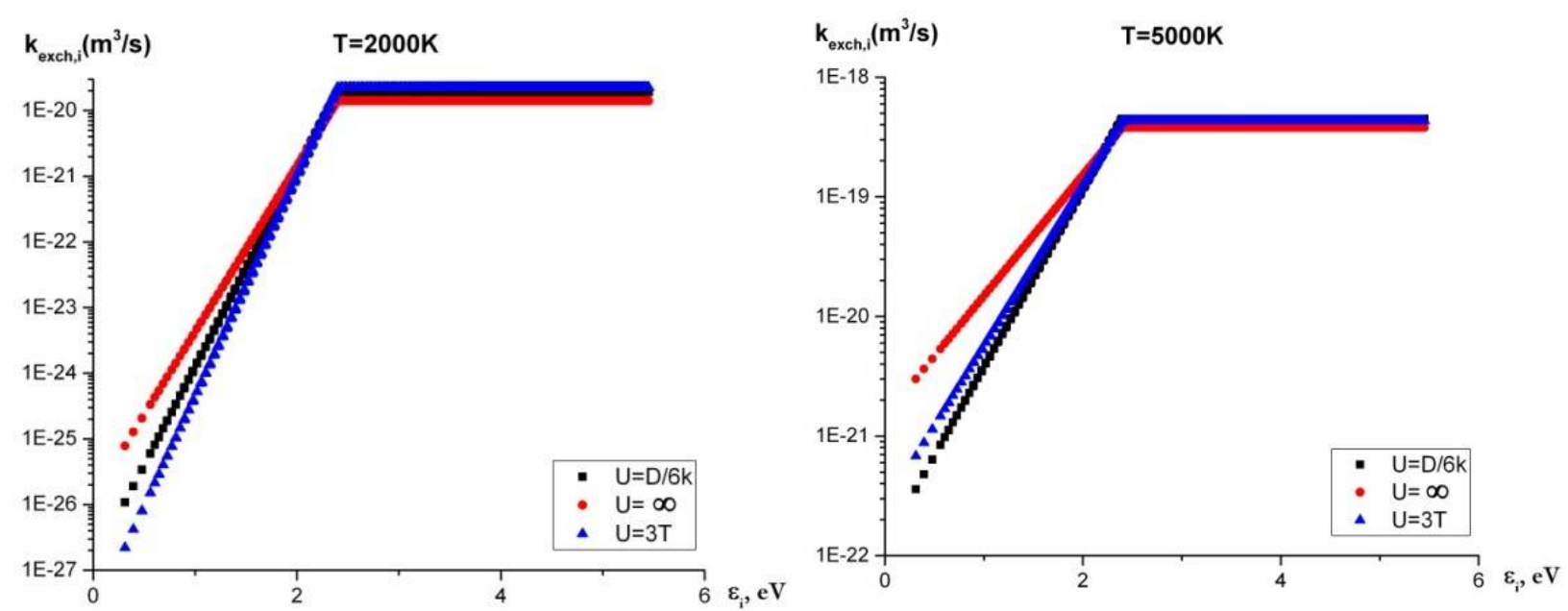

Рис. 3. Коэффициенты скорости обменных реакций как функция от колебательного уровня $\mathrm{CO}_{2}$ для различных значений параметра $U$ при различных температурах

\section{2. Многотемпературные коэффициенты скорости обменных реакций}

Многотемпературные коэффициенты скорости реакции могут быть получены осреднением поуровневых с различными колебательными распределениями

$$
k^{\text {exch }}\left(T, T_{1}, T_{2}, T_{3}, U\right)=\frac{1}{n} \sum_{i 1, i 2, i 3} n_{i 1 i 2 i 3}\left(T_{1}, T_{2}, T_{3}\right) k_{i}^{\text {exch }}(T, U)
$$

Здесь $T_{m}$ - колебательные температуры мод, $n_{i 1 i 2 i 3}$ - заселенности колебательных уровней, $n$ - числовая плотность газа.

В данной работе мы используем две модели: 1) двухтемпературную, в которой колебательные температуры различных мод равны между собой $\left(T_{1}=T_{2}=T_{3}=T_{\mathrm{v}}\right)$ и отличаются от температуры газа; 2) более реалистичную трехтемпературную модель, предложенную в [27]. Трехтемпературная модель основана на предположении о быстром резонансном VV обмене между симметричной и деформационной модой вследствие кратности их колебательных частот. Данный обмен ведет к выравниванию температур этих мод $\left(T_{1}=T_{2}=T_{12}\right)$, a температура антисимметричной моды $T_{3}$ отличается от $T_{12}$ из-за более медленных VV обменов с участием третьей моды. Основными каналами релаксации в данном случае является VT релаксация во второй и третье моде и VV обмены между ними.

Колебательные распределения для гармонических осцилляторов в данном случае имеют вид

$$
\begin{aligned}
& n_{i 1 i 2 i 3}\left(T_{12,} T_{3}\right)=\frac{n_{\mathrm{CO}_{2} s_{i 1 i 2 i 3}}}{Z_{v i b r}\left(T_{12,} T_{3}\right)} \exp \left(-\frac{i_{1} \varepsilon_{100}+i_{2} \varepsilon_{010}}{k T_{12}}-\frac{i_{3} \varepsilon_{001}}{k T_{3}}\right), \\
& Z_{v i b r}\left(T_{12,} T_{3}\right)=\sum_{i 1, i 2, i 3} S_{i 1 i 2 i 3} \exp \left(-\frac{i_{1} \varepsilon_{100}+i_{2} \varepsilon_{010}}{k T_{12}}-\frac{i_{3} \varepsilon_{001}}{k T_{3}}\right),
\end{aligned}
$$

где $n_{\mathrm{CO}_{2}}$ - числовая плотность молекул $\mathrm{CO}_{2} ; \varepsilon_{100}, \varepsilon_{010}, \varepsilon_{001}$ - колебательные энергии первых уровней соответствующих мод. При $T_{12}=T_{3}$ получаем двухтемпературную модель.

На рисунках 4 и 5 представлены неравновесные факторы (отношение многотемпературного коэффициента скорости реакции к соответствующему равновесному коэффициенту), полученные для двух- и трехтемпературной моделей при использовании различных значений параметра $U$ в поуровневой модели коэффициентов скорости обменных реакций. Значения колебательных температур взяты из работы [28] на различных расстояниях от фронта ударной волны при числах Маха 10 и 15. 


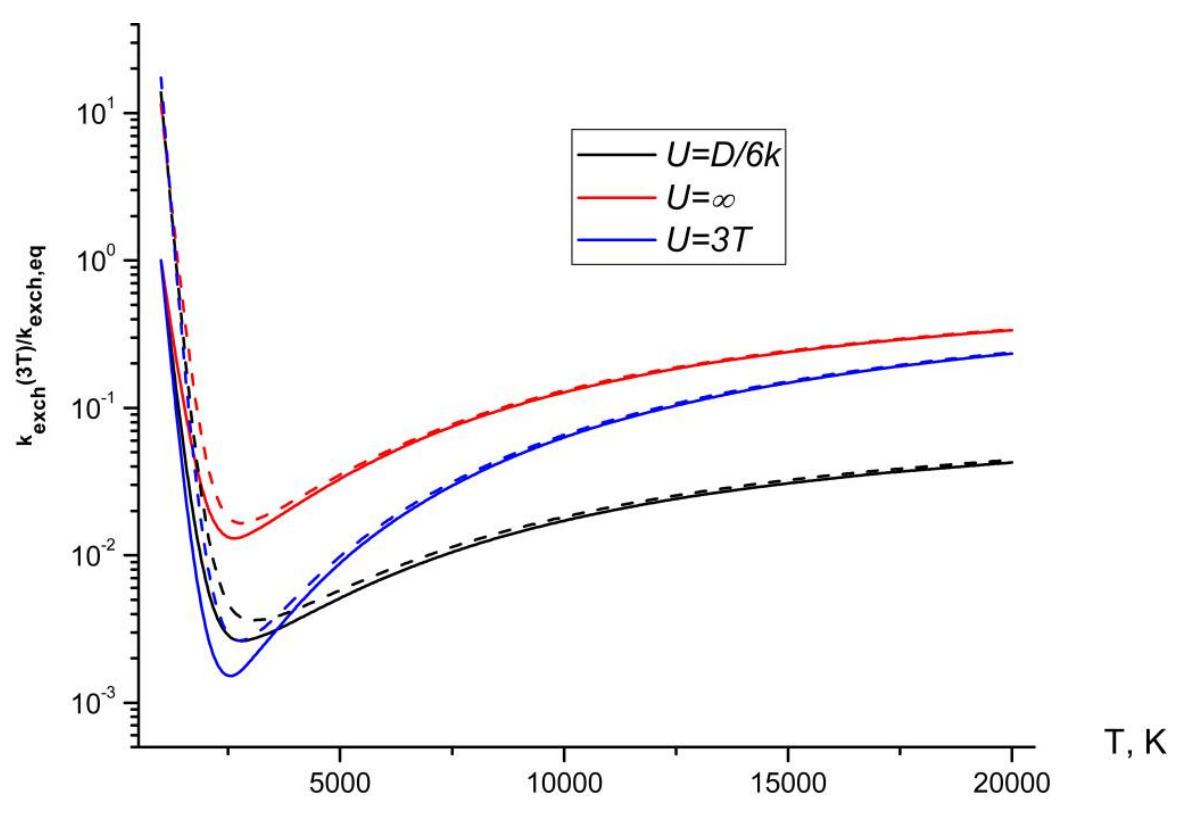

Рис.4. Неравновесные факторы для многотемпературных коэффициентов скорости обменной реакции. Сплошные кривые - двухтемпературная модель при $T_{v}=1000 \mathrm{~K}$. Штриховые кривые - трехтемпературная модель при $T_{12}=1000 \mathrm{~K}$ и $T_{3}=1400 \mathrm{~K}$

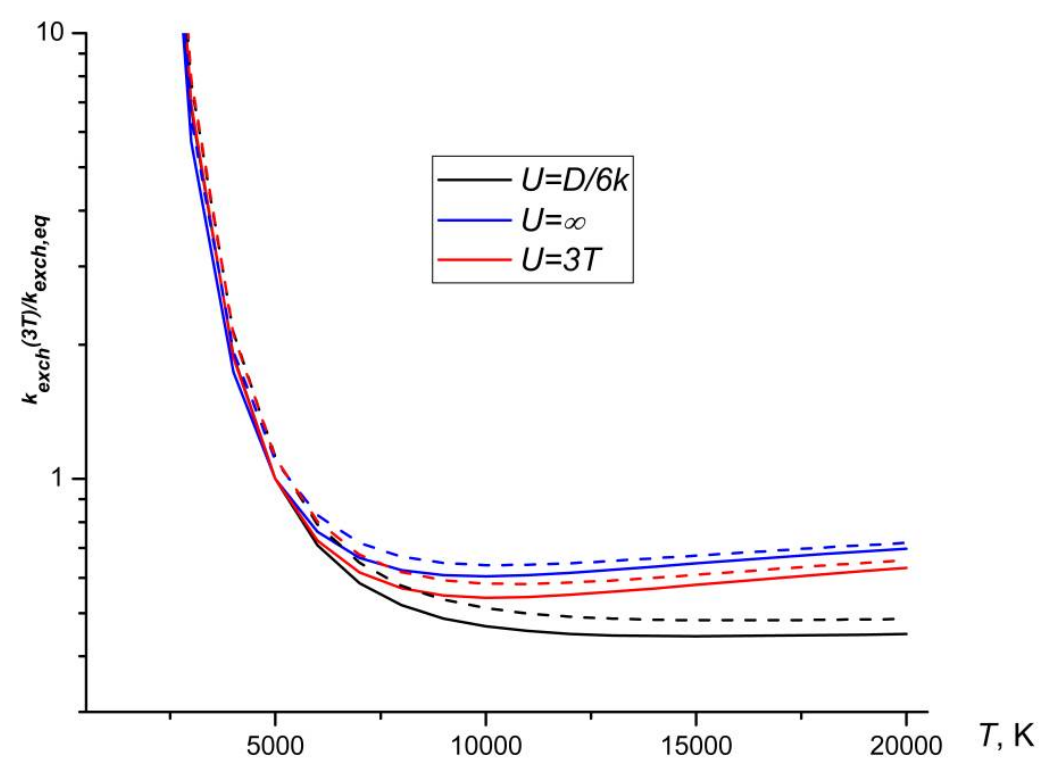

Рис. 5. Неравновесные факторы для многотемпературных коэффициентов скорости обменной реакции. Сплошные кривые - двухтемпературная модель при $T_{\mathrm{v}}=5000 \mathrm{~K}$. Штриховые кривые - трехтемпературная модель при $T_{12}=5000 \mathrm{~K}$ и $T_{3}=6100 \mathrm{~K}$

В рассмотренных условиях отличие коэффициентов скорости реакции, рассчитанных в двух- и трехтемпературных приближениях, невелико, поскольку разница колебательных температур за ударными волнами небольшая. Значительно более важным оказывается выбор параметра модели $U$. Интересно отметить ярко выраженный минимум в величине неравновесного фактора при низких колебательных температурах. 
Различие колебательных температур может оказывать более существенное влияние на скорость реакции в условиях, характерных для низкотемпературных плазменных технологий, когда для декомпозиции молекул углекислого газа проводится накачка колебательной энергии в антисимметричную моду.

\section{4. Заключение}

В работе предложены простые и эффективные с вычислительной точки зрения модели для расчета поуровневых коэффициентов скорости обменных реакций. Модель, построенная для реакций Зельдовича, обеспечивает хорошее согласие с квазиклассическими траекторными расчетами во всем рассмотренном диапазоне температур (от 1000 до $20000 \mathrm{~K}$ ) и колебательных энергий реагентов и продуктов. Данная модель может быть рекомендована для расчета неравновесных течений воздуха в условиях входа в атмосферу Земли. На основе модели, проверенной для воздуха, сделана первая попытка описания поуровневых обменных реакций в углекислом газе. Показано, что выбор параметра модели важен при вычислении коэффициента скорости реакции из нижних колебательных состояний. Рассчитаны многотемпературные коэффициенты скорости реакции для различных распределений. Показано, что для условий, характерных для ударно нагретого газа, выбор параметра модели более важен, чем учет различия температур колебательных мод молекулы $\mathrm{CO}_{2}$. Для условий, реализуемых при низкотемпературной конверсии $\mathrm{CO}_{2}$, влияние возбуждения отдельных мод на скорость реакции ожидается более значительным.

\section{Благодарности и ссылки на гранты}

Работа выполнена при поддержке гранта РФФИ № 18-01-00493 и НИР СПбГУ № 6.37.206.2016.

\section{Литература}

1. P.V. Marrone and C.E. Treanor. Chemical relaxation with preferential dissociation from excited vibrational levels // Phys. Fluids, 6(9):1215-1221, 1963.

2. Gilibert M., Aguilar A., Gonzales M., Sayos R. A quasiclassical trajectory study of the effect of the initial rovibrational level and relative translational energy on the dynamics of the $\mathrm{N}\left({ }^{4} S u\right)+\mathrm{O}_{2}\left({ }^{3} \Sigma_{g}^{-}\right) \rightarrow \mathrm{NO}\left({ }^{2} \Pi_{u}\right)+\mathrm{O}\left({ }^{3} P_{g}\right)$ atmospheric reaction on the ${ }^{2} \mathrm{~A}^{\prime}$ ground potential energy surface // Chem. Phys. 1993. Vol. 178. Pp. 287-303.

3. Gilibert M., Gimenez X., Gonzales M. et al. A comparison between experimental, quantum and classical properties for the $\mathrm{N}\left({ }^{4} S\right)+\mathrm{O}_{2}\left({ }^{3} \Sigma_{g}^{-}\right) \rightarrow \mathrm{NO}\left({ }^{2} \Pi\right)+\mathrm{O}\left({ }^{3} P\right)$ reaction // Chem. Phys. 1995. Vol. 191. Pp. 1-15.

4. Gilibert M., Gonzales M., Sayos R. et al. Reactive cross sections involving atomic nitrogen and ground and vibrationally excited molecular oxygen and nitric oxide // Molecular Physics and Hypersonic Flows / Ed. by M. Capitelli. Netherlands: Kluwer Acad. Publishers, 1996. Pp. 53-84.

5. Knab O., Frühauf H.H., Jonas S. Multiple temperature descriptions of reaction rate constants with regard to consistent chemical-vibrational coupling // AIAA paper 92-2947, AIAA 27th Thermophysics Conference. Nashville, USA,: July 1992. Pp. 1-15.

6. Knab O., Frühauf H.H., Messerschmid E.W. Theory and Validation of the Physically consistent Coupled Vibration-Chemistry-Vibration Model // J. Thermophys. Heat Transfer. 1995. Vol. 9, no. 2. Pp. 219-226.

7. Seror S., Druguet M.C., Schall E., Zeitoun D. A New Vibration-Exchange Reaction Coupling Model for Hypersonic Air Flows // AIAA Paper. 1997. Vol. 97-2556.

8. A. Aliat. State-to-state dissociation-recombination and chemical exchange rate coefficients in excited diatomic gas flows // Physica A, 387:4163-4182, 2008. 
9. Bose D., Candler G.V. Thermal rate constants of the $\mathrm{N}_{2}+\mathrm{O} \rightarrow \mathrm{NO}+$ reaction using ab initio ${ }^{3} \mathrm{~A}^{\prime \prime}$ and ${ }^{3}$ A' potential energy surfaces // J. Chem. Phys. 1996. Vol. 104, no. 8. P. 2825.

10. Bose D., Candler G.V. Thermal nonequilibrium rates of the Zeldovich reactions // AIAA Paper. 1997. Vol. 97-0133.

11. Birely J.H., Lyman J.L. Effect of reagent vibrational energy on measured reaction rate constants // J. Photochem. 1975. Vol. 4. P. 269.

12. STELLAR Database. http://esther.ist.utl.pt/pages/stellar.html

13. Luo Han, Kulakhmetov M., Alexeenko A. Ab initio state-specific $\mathrm{N}_{2}+\mathrm{O}$ dissociation and exchange modeling for molecular simulations // J. Chem. Phys. 2017. Vol. 146. P. 074303.

14. Esposito F., Armenise I. Reactive, inelastic and dissociation processes in collisions of atomic oxygen with molecular nitrogen // J. Phys. Chem. A. 2017. Vol. 121. Pp. 6211-6219.

15. E.V.Kustova and A.S.Savelev State-resolved models of chemical reactions for non-equilibrium flow simulations // 7th European Conference for Aeronautics and Aerospace Sciences (EUCASS). doi: 10.13009/EUCASS2017-111.

16. E.V. Kustova, A.S. Savelev, and O.V. Kunova Rate coefficients of exchange reactions accounting for vibrational excitation of reagents and products // AIP Conference Proceedings 1959, 060010 (2018); doi: $10.1063 / 1.5034671$.

17. М.Ю. Погосбекян, А.Л. Сергиевская Моделирование реакции диссоциации кислорода в термически неравновесных условиях: модели, траекторные расчеты, эксперимент // Химическая физика, 2018, том 37, № 4, с. 20-31. DOI: 10.7868/S0207401X18040039.

18. I.V. Arsentiev, B.I. Loukhovitski, A.M. Starik Application of state-to-state approach in estimation of thermally nonequilibrium reaction rate constants in mode approximation // Chemical Physics 398 (2012) 73-80

19. Armenise, I.; Kustova, E. State-to-state Models for $\mathrm{CO}_{2}$ Molecules: from the Theory to an Application to Hypersonic Boundary Layers // Chem. Phys. 2013, 415, 269-281.

20. Armenise, I.; Kustova, E. Mechanisms of Coupled Vibrational Relaxation and Dissociation in Carbon Dioxide // J. Phys. Chem. A. 2018, 122, 5107-120.

21. Armenise, I.; Kustova, E. Effect of Asymmetric Mode on CO2 State-to-State Vibrational-Chemical Kinetics // J. Phys. Chem. A. 2018, 122, 5107-120.

22. Fridman, A. Plasma Chemistry; Cambridge University Press: New York, 2008.

23. Kozak, T.; Bogaerts, A. Splitting of $\mathrm{CO}_{2}$ by Vibrational Excitation in Non-equilibrium Plasmas: a Reaction Kinetics Model // Plasma Sources Sci. Technol. 2014, 23, 045004.

24. Berthelot, A.; Bogaerts, A. Modeling of $\mathrm{CO}_{2}$ Splitting in a Microwave Plasma: How to Improve the Conversion and Energy Efficiency // J. Phys. Chem. C 2017, 121, 8236-8251.

25. Silva, T.; Grofulović, M.; Klarenaar, B.; Morillo-Candas, A.; Guaitella, O.; Engeln, R.; Pintassilgo, C.; Guerra, V. Kinetic Study of Low-temperature $\mathrm{CO}_{2}$ Plasmas under Non-equilibrium Conditions. I. Relaxation of Vibrational Energy // Plasma Sources Sci. Technol. 2018, 27, 015019.

26. Park C., Howe J.T., Howe R.L. et al. Review of Chemical-Kinetic Problems of Future NASA Missions, II: Mars Entries // J. Thermophys. Heat Transfer. 1994. Vol. 8, no. 1. Pp. 9-23.

27. Kustova, E.; Nagnibeda, E. On a Correct Description of a Multitemperature Dissociating CO2 Flow // Chem. Phys. 2006, 321, 293-310.

28. Kosareva Alena and Nagnibeda Ekaterina Multi-temperature models for shock heated flows of $\mathrm{CO} 2 / \mathrm{CO} / \mathrm{O}$ mixture // 7th European Conference for Aeronautics and Aerospace Sciences (EUCASS). doi: 10.13009/EUCASS2017-222. 